\title{
Rare Causes of Pleural Effusion in Children: Differential Diagnosis in a Pediatric Infectious Disease Clinic
}

\author{
Çocuklarda Plevral Efüzyonun Nadir Nedenleri: \\ Bir Çocuk Enfeksiyon Hastalıkları Kliniğinde Ayırıcı Tanı
}

\author{
Ayşe Kaman'(iD), Türkan Aydın Teke'(ID), Fatma Nur Öz'(iD), Sevgi Yaşar Durmuş'(iD), Rumeysa Yalçınkaya'(iD), \\ Gönül Tanır'(ID) \\ 'Clinic of Pediatric Infectious Diseases, Ankara Dr. Sami Ulus Maternity, Child Health and Diseases Training and Research Hospital, Ankara, Turkey
}

Cite this article as: Kaman A, Aydın Teke T, Öz FN, Yaşar Durmuş S, Yalçınkaya R, Tanır G. Rare causes of pleural effusion in children: differential diagnosis in a pediatric infectious disease clinic. J Pediatr Inf 2021;15(1):e26-e32.

Abstract

Objective: Exudative pleural effusion may occur during the course of many infectious and non-infectious diseases, and these diseases should be considered in the differential diagnosis of pleural effusion. In this study, we aimed to emphasize the rare causes and the importance of clinical and laboratory findings in the differential diagnosis of patients hospitalized with a preliminary diagnosis of parapneumonic effusion-empyema.

Material and Methods: In this study, the data of patients hospitalized with the preliminary diagnosis of parapneumonic effusion-empyema and who were diagnosed as a specific infection such as tuberculosis and parasitic diseases and as pleural effusion due to non-infectious causes in the following days were evaluated retrospectively.

Results: Between January 2010 and September 2019, a total of 13 patients [eight males (61.5\%), five females (38.5\%)], who were admitted with a preliminary diagnosis of parapneumonic effusion and pleural empyema, and subsequently received alternative diagnoses, were included in the study. Patients' complaints on admission were cough, fever, chest pain, abdominal pain, sputum, night sweats, weight loss and respiratory distress, respectively. There was no bacterial growth in blood and pleural fluid cultures of any patients. As a definitive diagnosis, tuberculosis was diagnosed in $5(38.4 \%)$ patients, lymphoma in $4(30.7 \%)$ patients, and hydatid cyst in $4(30.7 \%)$ patients. In one of the patients diagnosed with tuberculosis (20\%), Mycobacterium tuberculosis complex was detected by polymerase chain reaction while in $3(60 \%)$ patients by culture. Malignant cells were seen in pleural fluid cytology of 2 (15.4\%) patients. In two of the patients, a positive result was obtained by the indirect haemagglutination test for Echinococcus.
Öz

Giriş: Eksüdatif plevral efüzyon pek çok enfeksiyöz ve non-enfeksiyöz hastalıkların seyri sırasında görülebilir ve bu hastalarda ampiyem ve parapnömonik plevral efüzyonunun ayrıcı tanısı ayrıntılı olarak düşünülmelidir. Biz bu çalışmada parapnömonik efüzyon-ampiyem ön tanısı ile yatırılan hastaların ayrıcı tanısında nadir görülen nedenleri, klinik ve laboratuvar bulgularının önemini vurgulamayı amaçladık.

Gereç ve Yöntemler: Bu çalışmada kliniğimize parapnömonik efüzyon-ampiyem ön tanısı ile yatırılarak takipte tüberküloz ve paraziter hastalıklar gibi bir spesifik enfeksiyon ve enfeksiyon dışı nedenlere bağlı plevral efüzyon saptanan hastaların verileri geriye dönük olarak değerlendirildi.

Bulgular: Ocak 2010-Eylül 2019 tarihleri arasında parapnömonik efüzyon ve plevral ampiyem ön tanısı ile kabul edilip, sonradan alternatif tanılar alan ortalama yaşları $125.00 \pm 57.21$ ay olan $8^{\prime} \mathrm{i}(\% 61.5)$ erkek, $5^{\prime} \mathrm{i}$ (\%38.5) kız olmak üzere toplam 13 hasta çalışmaya dahil edildi. Başvuru şikayetleri sırasıyla öksürük, ateş, göğüs ağrısı, karın ağrısı, balgam, gece terlemesi, kilo kaybı ve solunum sıkıntısı idi. Kan kültürü ve plevral sıvı kültürü ile hastaların hiçbirinde bakteriyel üreme mevcut değildi. Kesin tanı olarak 5 (\%38.4) hastada tüberküloz, 4 (\%30.7) hastada lenfoma, 4 (\%30.7) hastada akciğer kist hidatiği tanısı konulmuştu. Tüberküloz tanısı alan hastalardan birinde (\%20) polimeraz zincir reaksiyonu ile, üçünde (\%60) mikobakteriyel kültürde Mycobacterium tuberculosis complex pozitif tespit edildi. 2 (\%15.4) hastanın plevral sıvı sitolojisinde malign hücre görüldü. Hastaların ikisinde kist hidatik indirekt hemaglütinasyon testinde pozitif sonuç elde edildi.

Sonuç: Çocukluk çağında eksüdatif plevral efüzyonun etyolojisinde en önemli neden parapnömonik efüzyon ve ampiyem olmakla birlikte, uy- 
Conclusion: Although parapneumonic effusion and empyema are the most important reasons in the etiology of exudative pleural effusion in childhood; tuberculosis, hydatid cyst and malignancy should be considered in pleural effusion that does not improve despite appropriate antibiotic treatment and drainage.

Keywords: Children, pleural effusion, malignancy, hydatid cyst, tuberculosis

\section{Introduction}

The pleural cavity is limited to two membranes, the visceral pleura covering the lung and the parietal pleura covering the chest wall and diaphragm. Normal fluids and proteins enter the pleural cavity through systemic circulation and are reabsorbed through parietal pleural lymphatics. During many diseases, apart from those caused by primary pleura, increased pleural effusion can be seen and classified as transudative or exudative according to the Light criteria considering the biochemical characteristic of the fluid (1). Transudative pleural effusion can be seen in children during the course of non-infectious systemic diseases such as hypoalbuminemia, congestive heart failure, cirrhosis, nephrotic syndrome, and myxedema. Parapneumonic effusion, which is exudative fluid collection, can be typically seen during the course of bacterial pneumonia, in $2-12 \%$ of all pneumonias, and in approximately $28 \%$ of pneumonias requiring hospitalization. Moreover, exudative pleural effusion can also be observed in viral (adenovirus, influenza virus), parasitic (cyst hydatid) and fungal infections, specific bacterial infections like tuberculosis, during the course of non-infectious diseases, such as malignancies, collagen vascular diseases, trauma or post-surgery, and as a side effect of drugs like hydralazine, amiodarone, procarbazine, and methotrexate, and it should be considered in the differential diagnosis of parapneumonic effusion and empyema (1-3). In this study, it was aimed to emphasize the importance of specific infections like tuberculosis or parasitic infections and malignancies and other rare causes in patients admitted to the pediatric infectious disease clinic with a preliminary diagnosis of parapneumonic effusion-empyema and underline the clinical and laboratory findings that pediatric infectious diseases specialists need to be mindful of.

\section{Materials and Methods}

Data of the patients admitted to the pediatric infectious disease clinic with a preliminary diagnosis of parapneumonic effusion-empyema and detected having pleural effusion due to a specific infection like tuberculosis and parasitic diseases and non-infectious causes during follow-up were retrospectively reviewed. Patients' age, sex, presenting symptoms and duration of symptoms, hemoglobin and total leucocyte (absolute neutrophil, lymphocyte, eosinophil, and monocyte) count, platelet count, erythrocyte sedimentation rate (ESR), C-reactive protein (CRP) and serum lactate dehydrogenase (LDH) levels, blood culture, pleural biochemical markers in patients in whom thorasynthesis had been made, adenosine gun antibiyotik tedavisi ve drenaja rağmen düzelmeyen, etkeni ortaya konulmamış plevral efüzyonda tüberküloz, kist hidatik ve malignite mutlaka akla gelmelidir.

Anahtar Kelimeler: Çocuk, plevral efüzyon, malignite, kist hidatik, tüberküloz

deaminase (ADA) if tested, Mycobacterium tuberculosis complex polymerase chian reaction (PCR) and culture, imaging results done for differential diagnosis, tuberculin skin test (TST), interferon gamma release assay (IGRA), cyst hydatid indirect hemaglutination (IHA) results, initial treatments started on the patients and final diagnoses were recorded.

Data were analysed using IBM SPSS Statistics. For descriptive analyses, mean \pm standard deviation was used for variables with normal distribution and median (minimum-maximum) was $p<0.05$.

\section{Results}

One hundred and sixty-one of the 1805 patients admitted to our hospital between January 2010-September 2019 for bacterial pneumonia (ICD code J13: Pneumonia, related to Streptococcus pneumoniae?, J14: Pneumonia, related to Haemophilus influenzae?, J15: bacterial pneumonias not classified elsewhere, J18: organisms not identified with pneumonia) were hospitalized with a preliminary diagnosis of parapneumonic effusion and empyema. A total of 13 patients, 8 boys (61.5\%) and 5 girls $(38.5 \%)$, who had received alternative diagnosis as final diagnosis were included into the study. Mean age of the patients was $125.00 \pm 57.21$ months, and median age was 120 months (minimum: 22 months, maximum: 203 months). The most common presenting symptoms were respectively found as cough in 8 patients (61.5\%), fever in 7 (53.8\%), chest pain in $6(46.2 \%)$, abdominal pain in 3 (23.1\%), expectorating in 3 (23.1\%), night sweating in $3(23.1 \%)$, weigh loss in $3(23.1 \%)$, and respiratory distress in 1 (7.7\%) patient. Table 1 shows age, sex, presenting symptoms and physical examination findings of the patients. Patients' median lenght of complaints prior to presentation to hospital was 10 days (minimum: 1 day, maximum: 60 days), and in patients with fever, median length of fever was 7 days (minimum: 3 days, maximum: 30 days). Patients' mean hemoglobin $(\mathrm{Hb})$ concentration was $12.53 \pm 1.88$ (median; 11.9, range; 10.0-16.3) $\mathrm{g} / \mathrm{dL}$, peripheral white blood cell count (WBC); $9935 \pm 3449$ (median; 9500, range; 5820-16300) $/ \mathrm{mm}^{3}$, platelet count; $514.076 \pm 202113$ (median; 505000, range; 165000837000)/mm³ $\mathrm{mm}^{3}$, $93.04 \pm 63.41$ (median; 97, range; 13.6-187) $\mathrm{mg} / \mathrm{L}, \mathrm{ESR} ; 64.76 \pm 30.65$ (median; 61, range; 12-125) mm/h. Table 1 summarizes patients' laboratory findings. All patients underwent thorasynthesis on their admission to hospital, and thorasynthesis findings were evalutaed as pleural empyema in 11 patients (84.6\%) and parapneumonic pleural effusion in 2 patients (15.4\%). Table 2 shows patients' pleural fluid cell 


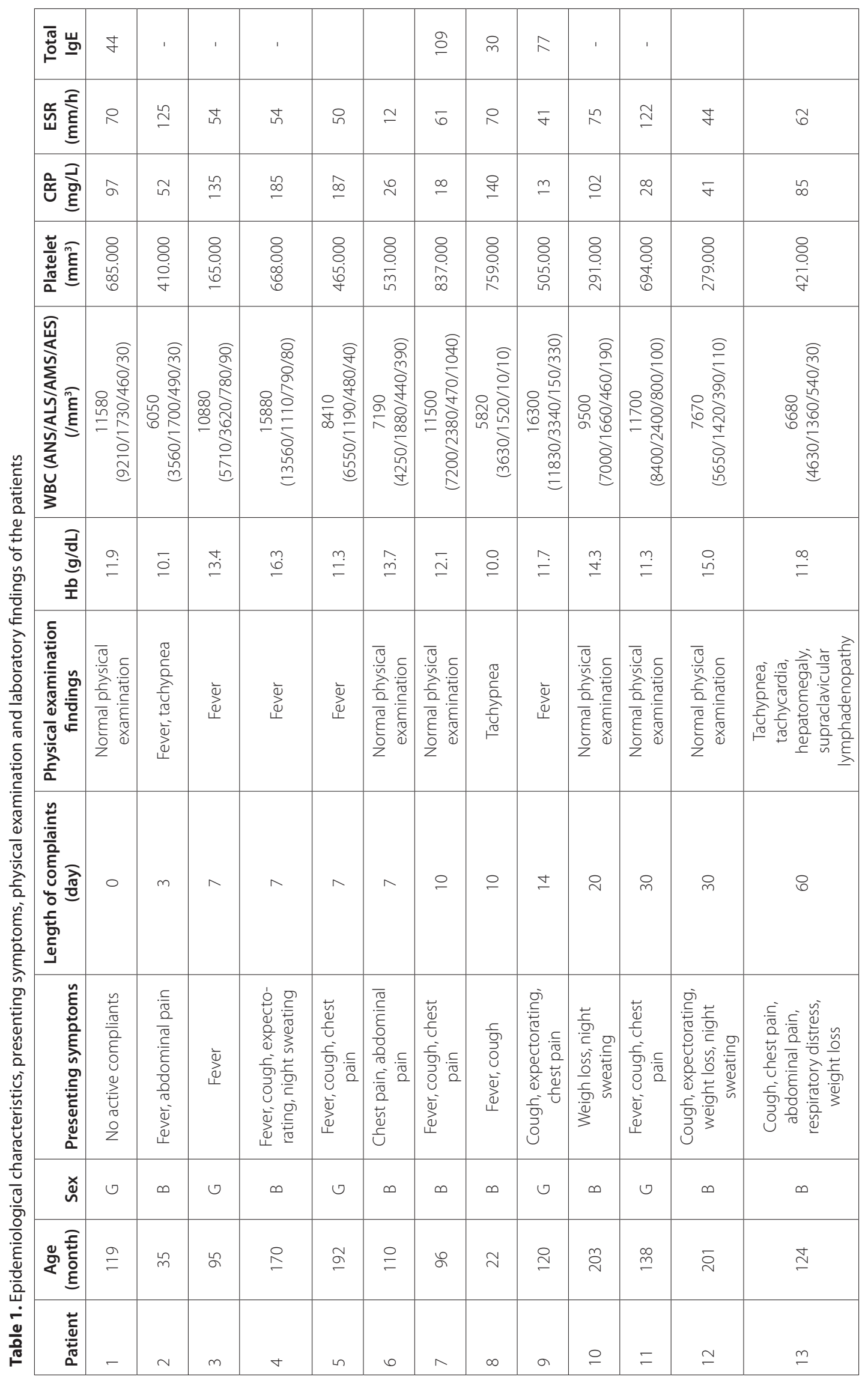


Kaman et al.

\begin{tabular}{|c|c|c|c|c|c|c|c|c|c|c|c|c|c|}
\hline 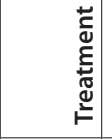 & 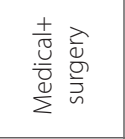 & $\begin{array}{l}\overline{\bar{J}} \\
\overline{\bar{g}} \\
\sum\end{array}$ & $\begin{array}{l}\overline{\bar{g}} \\
\overline{\bar{g}} \\
\sum\end{array}$ & 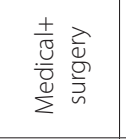 & $\begin{array}{l}\overline{\bar{J}} \\
\overline{\bar{O}} \\
\sum\end{array}$ & $\begin{array}{l}\overline{\widetilde{J}} \\
\overline{\bar{g}} \\
\sum\end{array}$ & 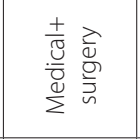 & $\begin{array}{l}\overline{\widetilde{J}} \\
\overline{\bar{\sigma}} \\
\sum\end{array}$ & 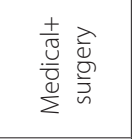 & $\begin{array}{l}\overline{\bar{J}} \\
\frac{\widetilde{J}}{\sum}\end{array}$ & $\begin{array}{l}\overline{\widetilde{J}} \\
\overline{\bar{g}} \\
\sum\end{array}$ & $\begin{array}{l}\overline{\bar{\sigma}} \\
\frac{\tilde{O}}{\bar{d}} \\
\sum\end{array}$ & $\begin{array}{l}\frac{\overline{\widetilde{J}}}{\bar{\sigma}} \\
\sum \\
\sum\end{array}$ \\
\hline 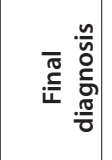 & 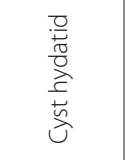 & 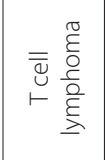 & 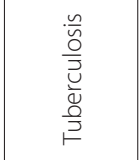 & 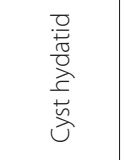 & 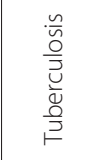 & 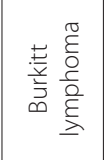 & 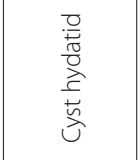 & $\begin{array}{l}\frac{n}{\overline{0}} \\
\frac{0}{3} \\
\frac{\tilde{u}}{\tilde{N}} \\
\frac{0}{p}\end{array}$ & 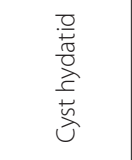 & 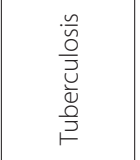 & 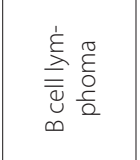 & $\begin{array}{l}\frac{n}{\overline{0}} \\
\frac{0}{\bar{n}} \\
\frac{\overline{0}}{\pi} \\
\frac{0}{2}\end{array}$ & 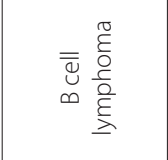 \\
\hline $\begin{array}{l}\frac{d}{d} \\
\stackrel{5}{5} \\
\stackrel{5}{⺊}\end{array}$ & $I^{\prime}$ & 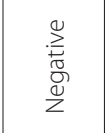 & 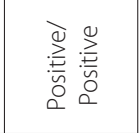 & 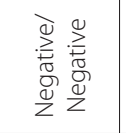 & 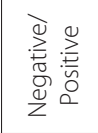 & 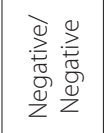 & 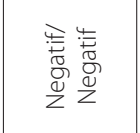 & 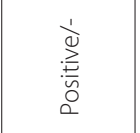 & 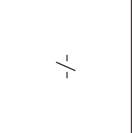 & 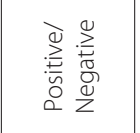 & 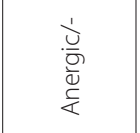 & 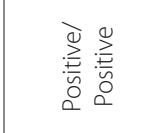 & 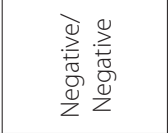 \\
\hline 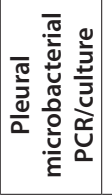 & $I^{\prime}$ & 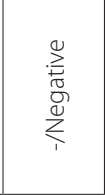 & 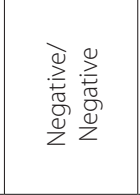 & 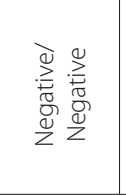 & 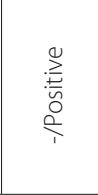 & 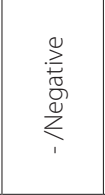 & 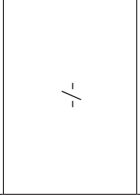 & 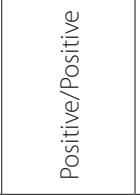 & $\frac{1}{i}$ & 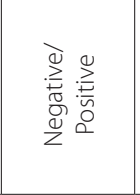 & 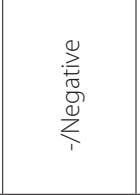 & 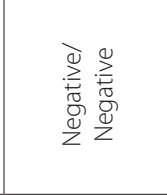 & 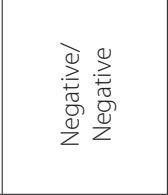 \\
\hline 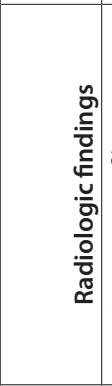 & 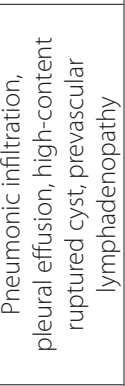 & 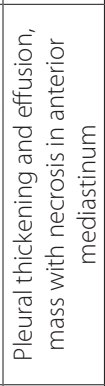 & 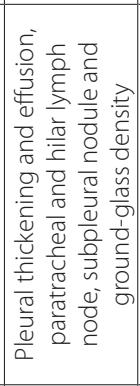 & 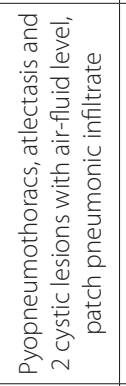 & 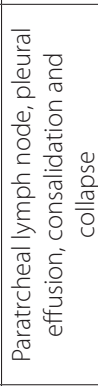 & 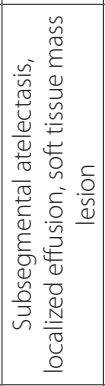 & 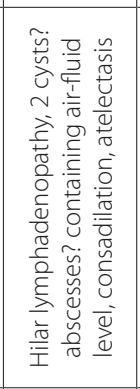 & 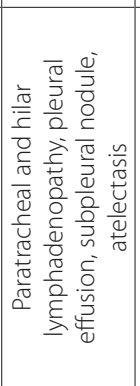 & 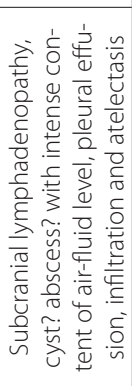 & 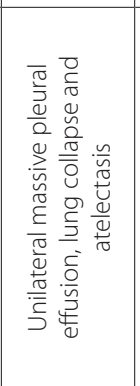 & 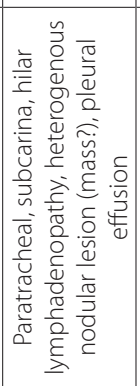 & 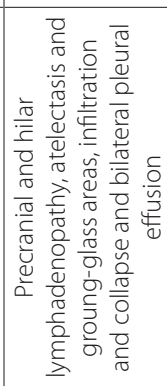 & 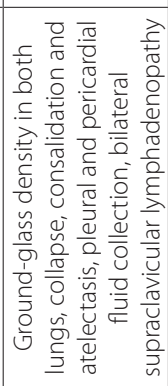 \\
\hline 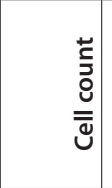 & 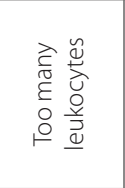 & 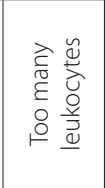 & 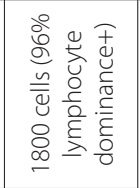 & 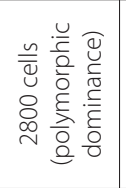 & 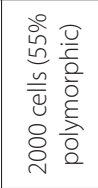 & 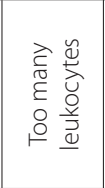 & 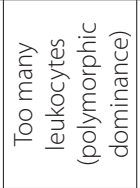 & 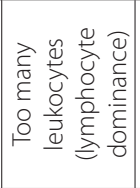 & 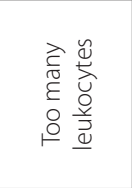 & 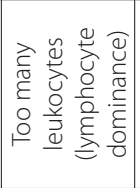 & 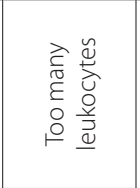 & 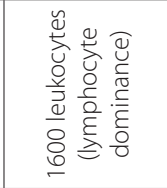 & 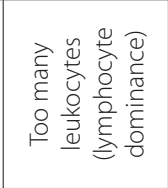 \\
\hline $\begin{array}{l}\bar{\sigma} \\
\text { 竞 } \\
\frac{\omega}{\alpha}\end{array}$ & ' & ' & ' & , & 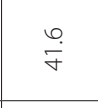 & ' & , & $\stackrel{n}{i}$ & & 芦 & 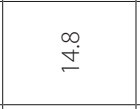 & $\stackrel{\infty}{m}$ & $\stackrel{m}{m}$ \\
\hline 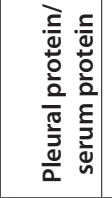 & $\stackrel{\hat{q}}{\stackrel{m}{q}}$ & $\begin{array}{l}n \\
\stackrel{n}{0} \\
\stackrel{0}{0} \\
\stackrel{+}{+}\end{array}$ & 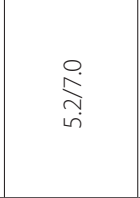 & 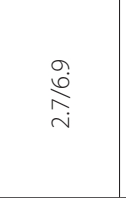 & 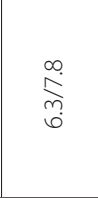 & 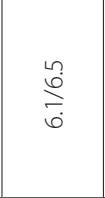 & ' & 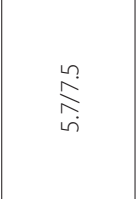 & $\underset{\substack{0\\
}}{\stackrel{n}{n}}$ & $\begin{array}{l}0 \\
\infty \\
\text { fo } \\
\text { fin }\end{array}$ & $\underset{i n}{\stackrel{+}{*}}$ & 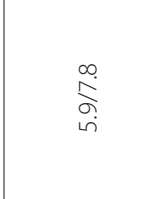 & $\begin{array}{l}\stackrel{0}{\dot{a}} \\
\stackrel{a}{\sigma}\end{array}$ \\
\hline 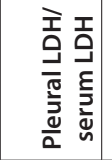 & 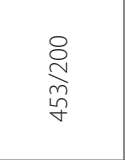 & 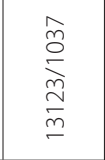 & 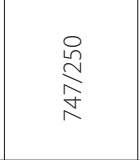 & $\begin{array}{l}\stackrel{2}{1} \\
\stackrel{n}{2} \\
\stackrel{2}{=}\end{array}$ & 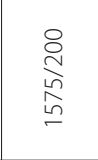 & 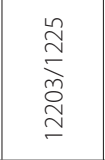 & & 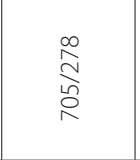 & $\begin{array}{l}\text { ळे } \\
\stackrel{0}{0}\end{array}$ & 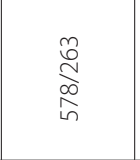 & 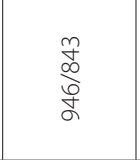 & 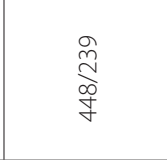 & 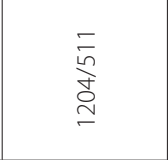 \\
\hline 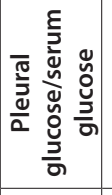 & $\begin{array}{l}\hat{a} \\
\hat{0}\end{array}$ & $\frac{ \pm}{\stackrel{亠}{\alpha}}$ & 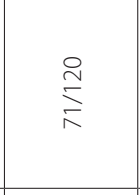 & $\underset{i n}{\stackrel{n}{\Sigma}}$ & ฟे & $\begin{array}{l}\infty \\
\underset{\sim}{\infty}\end{array}$ & & 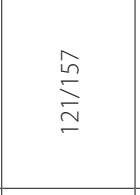 & $\underset{m}{\stackrel{\infty}{f}}$ & $\begin{array}{c}\infty \\
\text { Oे } \\
\text { ô }\end{array}$ & $\begin{array}{l}\stackrel{\circ}{\sigma} \\
\text { }\end{array}$ & $\underset{\infty}{\stackrel{\infty}{\infty}}$ & $\stackrel{\alpha}{\Omega}$ \\
\hline 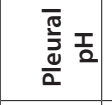 & $\stackrel{\text { g. }}{N}$ & $\underset{n}{\Uparrow}$ & 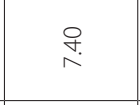 & ใู & 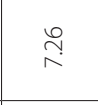 & $\stackrel{\infty}{\pi}$ & $\stackrel{\circ}{\wedge}$ & 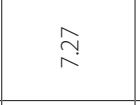 & $\bar{\curvearrowright}$ & $\stackrel{\circ}{\wedge}$ & $\stackrel{n}{\curvearrowright}$ & 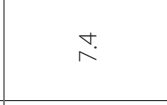 & $\stackrel{\circ}{\wedge}$ \\
\hline 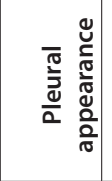 & 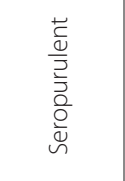 & $\begin{array}{l}\frac{\vec{c}}{\frac{\omega}{v}} \\
\frac{2}{2} \\
\frac{2}{2}\end{array}$ & 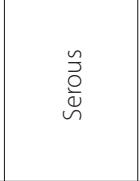 & 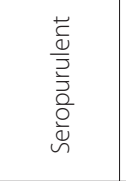 & $\begin{array}{l}\stackrel{n}{0} \\
\stackrel{\bar{\nu}}{n}\end{array}$ & 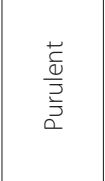 & $\begin{array}{l}\frac{\vec{c}}{\frac{0}{2}} \\
\frac{\bar{v}}{2} \\
\frac{2}{2}\end{array}$ & $\begin{array}{l}\frac{\tilde{a}}{0} \\
\stackrel{\bar{v}}{\sim}\end{array}$ & $\begin{array}{l}\stackrel{u}{\vec{d}} \\
\stackrel{\bar{d}}{\sim}\end{array}$ & $\begin{array}{l}\stackrel{n}{0} \\
\stackrel{\bar{d}}{\sim}\end{array}$ & $\begin{array}{l}\stackrel{n}{\bar{v}} \\
\stackrel{\bar{v}}{\sim}\end{array}$ & 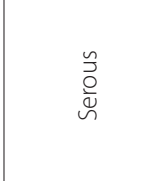 & 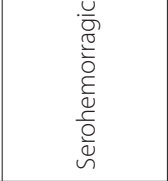 \\
\hline 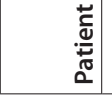 & - & $\sim$ & $m$ & $\nabla$ & in & 0 & $\wedge$ & $\infty$ & $a$ & $\stackrel{ }{\circ}$ & $=$ & $\simeq$ & $\stackrel{m}{n}$ \\
\hline
\end{tabular}


count, gram culture and results of biochemical analyses. While a negative and positive result was determined in 6 (54.5\%) and $4(36.3 \%)$ of the patients respectively among the patients in whom tuberculin skin test $(n=11)$ was performed, no enduration was seen in one patient (9\%) and was interpreted as TST anergic. Interferon gamma release assay yielded a negative result in five patients $(62.5 \%)$ and positive result in three $(37.5 \%)$ among the patients tested $(n=8)$. History of contact with a tuberculosis patient was present in three patients $(23.1 \%)$ epidemiologically. Blood culture and pleural fluid culture were taken from all patients, and no growth was seen in any of them. In 1 (20\%) of the patients diagnosed with tuberculosis $(n=5), M$. tuberculosis complex was deterimined positive in the pleural fluid with PCR; however, M. tuberculosis complex growth was observed in the mycobacterial culture of the pleural culture of three patients (60\%). Pleural ADA level was studied in 4 of the patients with confirmed tuberculosis $(n=5)$, and ADA level was found as $>40 \mathrm{IU} / \mathrm{L}$ in 3 patients $(75 \%)$. Malignant cells were seen in the pleural fluid cytology of 2 patients (15.4\%). Bone marrow aspiration was performed in 4 patients (30.8\%), and no malignant infiltrations were confirmed. Blood was taken for IHA test in 3 patients (23.1\%) due to cyst hydatid, and a positive result was obtained in two patients. All patients underwent contrast-enhanced thoracic computed tomography (CT). Right lung involvement was present in 7 patients (53.8\%), left lung involvement in five (38.5\%) and bilateral involvement in 1 patient (7.7\%). Respectively, radiological findings were as follows: pleural effusion in 13 patients (100\%) (massive effusion in 1 patient), atelectasis in eight patients $(61.5 \%)$, intrathoracic lymphadenopathy in patological sizes in 8 patients (61.5\%), pneumonic infiltration-consolidation in 7 patients (53.8\%), cystic lesions in the lung in 4 patients $(30.8 \%)$, lung collapse in five patients (\%), abscess-like lesion containing air-fluid level in the lung in 3 patients (23.1\%), mass lesion in the lung in 3 patients (23.1\%), ground-glass density in 3 patients (23.1\%), and subpleural nodule appearance in 2 patients (15.4\%). Tube thoracostomy or underwater seal drain with intrapleural catheter was performed in $10 \mathrm{pa}-$ tients (76.9\%). As empirical antibiotic treatment, 11 patients (84.6\%) were started on non-pseudomonal third generation cephalosporin, 2 patients (15.45) were started on beta-lactam antibiotics combined with beta-lactamase inhibitor, 7 patients (53.8\%) received clindamycin, 5 patients receieved (38.5\%) macrolide, and 4 patients (30.8\%) were given glycopepide antibiotics. During follow-up, 1 (7.7\%) patient's treatment was changed to anti-pseudomonal penicillin and two (15.4\%) patients' treatment was changed to carbapenem. As definitive diagnosis, 5 patients (38.4\%) were diagnosed with tuberculosis, 4 (30.7\%) with lymphoma (1 patient with B cell lymphoma, 2 with T cell lymphoma, 1 with Burkitt lymphoma), and 4 (30.7\%) with lung cyst hydatid. In one of the patients determined to have lung cyst hydatid, a lesion compatible with left renal cysts was also present. While four patients with cyst hydatid were surgically treated, two of the patients diagnosed with malignancies and two with cyst hydatid were referred to another center.

\section{Discussion}

Pleural diseases and pleural effusion can develop in the course of various diseases. Age distribution of the patients, evaluation time periods, and the epidemiologic characteristics of the region the study is carried out are important for etiological studies. Pneumonia, malignant effusion, pulmonary embolism, and gastrointestinal system-related diseases constitute approximately $90 \%$ of exudative pleural effusion $(1,4-$ 6). In a study including 3077 patients with pleural effusion in Portugal, effusion reasons have been reported as malignant effusion (27\%) followed by heart failure $(21 \%)$, pneumonia (19\%), tuberculosis (9\%), abdominal surgery (4\%), pericardial disease (4\%), and cirrhosis (3\%) (7). During childhood, pleural effusion is mostly observed in the course of infectious diseases of the lung (8). Pleural effusion caused by reasons other than lung disease that can be more frequently seen in adults is rare in children; however, intrathoracic or metastatic malignancies, heart failure and rheumatological diseases can lead to pleural effusion during childhood. Malignancy-related pleural effusion, which is one of the most common reasons of exudative pleural effusion in adults, is quite rare in children $(4,8)$. Patients with malignant pleural effusion can be asymptomatic but they also can present with empyema-like clinical symptoms such as fever, wheezing, cough, and respiratory distress $(1,8)$. Localized or not-fully localized chest pain is seen in $60 \%$ of the patients (9). In malignant effusions, exudative pleural fluid is seen with lymphocyte dominance. $\mathrm{pH}$ and glucose can be low in the fluid, and it has been reported to be associated with the advanced stage of the malignancy $(1,9)$. LDH, an intracellular enzyme, can be a marker for cell turn-over in the pleural fluid and increases in all exudative fluids (1). Serum LDH activity increases in many diseases including leukemia and lymphoma. Therefore, it can be used as a significant marker in the clinical diagnosis of the malignancy, monitoring tumor size after treatment and determining prognosis (10). Thus, high serum LDH levels can be a warning in terms of malignancy. We had no patients diagnosed with primary lung tumor or pleural tumor; however, four of our patients were diagnosed with intrathoracic lymphoma. Among these four patients, while three had chest pain and one had abdominal pain, a pathological finding could not be found on physical examination in two patients. While pleura pH was below the limit of 7.3 in four of our patients with malignancies, pleura glucose was below the normal level in only two patients. While pleural protein levels of these patients were not so high, that pleural LDH and serum $L D H$ levels were higher than the rest of the patients was a warning sign.

First findings of pulmonary cyst hydatid resulting from the attachment of Echinococcus granulosus larvae, which is dog te- 
nia, onto the lungs are mostly caused by lesions that suppress the surrounding tissues, and the most frequently seen clinical findings could include cough, chest pain, respiratory distress, and hemoptysis $(11,12)$. Infected pulmonary cyst hydatid may show radiological and clinical findings similar to a lung abscess. It may lead to chemical pneumonia in the surrounding parenchymal tissue and accompanying pleural effusion with the rupture of the cyst or tension pneumothorax, effusion and even empyema with the opening of the cyst to the pleural cavity (11-13). In a retrospective study including 120 (64 boys, 56 girls) children with a mean age of $10.15 \pm 3.93$ years followed with the diagnosis of pulmonary cyst hydatid between 20072012 in our country, $34.2 \%$ of the patients have been reported to have presented with ruptured cyst hydatid. In addition, it has been reported that $8.3 \%$ of all patients $(n=10)$ underwent tube thoracostomy with empyema diagnosis and pulmonary cyst hydatid diagnosis was made in these patients by surgical exploration due to the fact that recovery took long in follow-up (11). In a retrospective study from Greece including 187 pediatric patients aged 2-14 years followed for cyst hydatid in a period of 39 years, lung involvement has been reported in 96 patients $(51.3 \%)$. It has been established that $53.1 \%$ of the patients diagnosed with pulmonary cyst hydatid were asymptomatic, and the most common clinical findings were cough, chest pain and fever, respectively. In only 3 patients with complicated cyst hydatid, development of pleural empyema and hydrothorax has been reported (14). In a study from our country including 11 cyst hydatid patients, with a mean age of $35.33 \pm 17.52$ months, who were diagnosed differently with atypical clinical and radiological characteristics in a 3-year period, the most frequently encountered symptoms have been reported as chest pain (81.8\%) and cough (72.7\%) and the most commonly detected radiologic findings (27.27\%) have been demonstrated as lobar pneumonic infiltration, nodule in the lung parenchyma and pleural thickening and pleural fluid. Preliminary diagnoses were respectively parapneumonic effusion and empyema (45.4\%), lung cancer (27.2\%), lung abscess $(18.1 \%)$, and congenital cystic adenomatoid malformation (9\%). It has been reported that 3 patients considered to have empyema and parapneumonic effusion had pleural fluid bacterial growth (1 Pseudomonas aeruginosa, 1 Streptococcus pneumoniae, 1 Staphylococcus aureus), and the author has emphasized that cyst hydatid can frequently mimic other pulmonary diseases and its atypical clinical and radiological appearance may cause misdiagnosis (15). While only 1 patient out of 4 who were diagnosed with pulmonary cyst hydatid was asymptomatic, the other patients presented with fever, cough, and chest pain. While the physical examination of the two patients was completely normal, the only pathological finding detected in the other 2 patients was fever. Pleural fluid of all patients was exudative, and elevated serum eosinophil was confirmed in only 1 patient. Positive result supportive of the diagnosis was obtained in 2 of the 3 patients tested for cyst hydatid IHA. Cystic lesion with high content and air-fluid level was seen in all patients on computed tomography, and it was a warning for complicated cyst hydatid.

Tuberculosis is the most common reason of deaths related to single infectious agent worldwide. Due to the fact that tuberculosis is still frequently seen in our country, tuberculous pleurisy must be kept in mind in every patient presenting with pleural effusion that does not ameliorate with appropriate antibiotic treatment and drainage and/or whose etiology is unknown $(9,16)$. Pleural effusion at a rate of $12-38 \%$ can be seen in untreated pulmonary tuberculosis in childhood, and it is concomitant with a pulmonary parenchymal focus in 40$50 \%$ of the children. Diagnosis is rather difficult since it is a challenge to detect the bacillus in the pleural cavity, and other reasons for pleural effusion and specifically malignancies must be kept in mind in differential diagnosis (9,16-18). In a study including 112 pediatric patients with pleural tuberculosis in China, mean age of the patients and the most common symptoms have been reported as $11.6 \pm 3.2$ years and fever, dyspnea and cough, respectively. In all patients, the pleural fluid was exudative and lymphocytes were abundant in $91.2 \%$, ADA level was high (> 40U/L) in $74.5 \%$, and M. tuberculosis was detected in the pleural culture of $36.6 \%$. Involvement concomitant with pulmonary parenchymal focus was detected in $35.7 \%$ of the patients, and bilateral effusion was only detected in $3.6 \%$ (19). In a study analyzing the data of a pediatric tuberculosis clinic between the years 1984 and 2008 in the USA, pleural tuberculosis has been confirmed in 5.3\% $(n=45)$ of the 860 children with tuberculosis. The diagnosis of pleural tuberculosis has been confirmed by radiological findings $(100 \%)$, positive TST $(89 \%)$, positive culture $(56 \%)$, histopathology (27\%) and PCR (7\%). Involvement together with pulmonary parenchymal focus (infiltration, hilar or mediastinal lymphadenopathy) has been detected in $71.1 \%$ of the patients (17). In another study from our country including pediatric patients followed for pleural tuberculosis, 7 children out of 65 diagnosed with tuberculosis have been reported having pleural tuberculosis. It has been reported that isolated pleural involvement was seen in $57.1 \%$ of the patients, and ADA elevation (> 40IU/L) and lymphocyte dominant pleural pleocytosis were present in $85.7 \%$. It has been emphasized that elevated ADA is supportive of tuberculosis in the presence of lymphocyte-rich pleural effusion (18). In our study, while only 1 of the patients confirmed having tuberculosis ( 2 girls, 3 boys) was 2 years old, the other patients were older than 8 years. The most common complaints of our patients were fever, cough, weight loss, and night sweating. Unilateral involvement and concomitant involvement with a pulmonary tuberculosis focus were seen in most of the patients ( $80 \%$ ). While positive results were obtained by TST and IGRA in all patients with confirmed tuberculosis diagnosis, ADA was detected in 1 patient as <40IU/L 
and polymorphic dominance was detected in the pleural fluid of 1 patient, and diagnosis was confirmed with mycobacterial culture and IGRA results in these patients. Microbiologically, M. tuberculosis was detected positive in most of the patients.

In conclusion, the most important cause in the etiology of exudative pleural effusion in childhood is parapneumonic effusion and empyema, and tuberculosis, cyst hydatid and malignancies must be brought to mind in pleural effusion that does not ameliorate with appropriate antibiotic treatment and drainage and/or whose agent has not been determined. In the presence of lymphocyte dominance in the pleural fluid, ruling out malignancies with pleural cytology should be considered. In patients with complaints such as long-lasting fever, weight loss, and night sweating, TST, IGRA, and pleural ADA levels, along with the cell distribution of the pleural fluid, can be used to support tuberculosis diagnosis. Advanced imaging methods like contrast-enhanced thoracic computed tomography can be useful both in surgery and in identifying the underlying disease in empyema differential diagnosis.

Ethics Committe Approval: The approval for this study was obtained from T.C. Ministry of Health Ankara Dr. Sami Ulus Maternity, Child Health and Diseases Training and Research Hospital Ethical Committee (Decision No: 2019/10 Date: 10.10.2019).

Informed Consent: Patient consent was obtained.

Peer-review: Externally peer-reviewed.

Author Contributions: Concept- AK, FNÖ, GT; Design- TAT, SYD; Supervision- TAT, GT; Resource-AK, RY; Data Collection and/or Processing- AK, RY, SYD; Analysis and /or Interpretation- AK, TAT, FNÖ; Literature Search- GT, AK, Writing- AK, TAT; Critical Review- TAT, FNÖ, GT.

Conflict of Interest: Authors declared no conflict of interest.

Financial Disclosure: The authors declared that this study has received no financial support.

\section{References}

1. Broaddus VC, Light RW. Pleural effusion. In: Broaddus VC, Mason RJ, Ernst JD, King TE, Lazarus SC, Murray JF, Nadel JA, Slutsky AS, Gotway MB (eds). Murray and Nadel's Textbook of Respiratory Medicine. 6th ed. Philadelphia, PA: Elsevier; 2016: 1396-424. [CrossRef]

2. Mani CS. Acute pneumonia and its complications. In: Long SS, Ficher M, Prober CG (eds). Principles and Practice of Pediatric Infectious Diseases. $5^{\text {th }}$ ed. Philadelphia, PA: Elsevier; 2018; 239-49. [CrossRef]
3. Maskel NA, Light RW. Pleural infections. In: Broaddus VC, Mason RJ, Ernst JD, King TE, Lazarus SC, Murray JF, Nadel JA, Slutsky AS, Gotway $M B$ (eds). Murray and Nadel's Textbook of Respiratory Medicine. $6^{\text {th }}$ ed. Philadelphia, PA: Elsevier; 2016: 1425-38. [CrossRef]

4. Göğüs Hastalıkları Uzmanları için Plevra Hastalıkları Tanı ve Tedavi Rehberi. Ankara, TTD ve TÜSAD Yayını; 2016: 1-3. [CrossRef]

5. Prabhudesai PP, Mahashur AA, Mehta N, Ajay R. Exudative pleural effusions in patients over forty years of age--an analysis of seventy-six patients. J Postgrad Med 1993;39:190-3. [CrossRef]

6. Hooper C, Lee YC, Maskell N; BTS Pleural Guideline Group. Investigation of a unilateral pleural effusion in adults: British Thoracic Society Pleural Disease Guideline 2010. Thorax 2010;65 Suppl 2:ii4-17. [CrossRef]

7. Porcel JM, Esquerda A, Vives M, Bielsa S. Etiology of pleural effusions: analysis of more than 3,000 consecutive thoracosenteses. Archivos de Bronconeumoligia 2014;50:161-5. [CrossRef]

8. Özek, G., Uçan, B., Girit, S., Hoşgör, M., Cartı, Ö., Can, D., \& Diniz, G. Plevral efüzyonun nadir nedenleri: Iki olgu sunumu. Izmir Dr. Behçet Uz Çocuk Hastanesi Dergisi 1: 84-7. [CrossRef]

9. Karkhanis VS, Joshi JM. Pleural effusion: diagnosis, treatment, and management. Open Access Emerg Med 2012;4: 31-52. [CrossRef]

10. Yavuz D. Hodgkin Lenfomalı Hastalarda Tedavi Öncesi ve Sonrası Laboratuvar Değerleri ile Kemoterapi ve Radyoterapinin Kardiyopulmoner Fonksiyonlar Üzerine Etkisi. Tıpta Uzmanlık Tezi. İstanbul Eğitim Araştırma Hastanesi. 2009. [CrossRef]

11. Cevik M, Boleken ME, Kurkcuoglu IC, Eser I, Dorterler ME. Pulmonary hydatid disease is difficult recognized in children. Pediatr Surg Int 2014;30:737-41. [CrossRef]

12. Santivanez S, Garcia HH. Pulmonary cystic echinococcosis. Curr Opin Pulm Med 2010;16:257-61. [CrossRef]

13. Haberal MA, Akar E, Dikis OS, Kaya M. Surgical treatment of childhood pulmonary hydatidosis: an analysis of 25 cases. Tanaffos 2018;17:2804. [CrossRef]

14. Petropoulos AS, Chatzoulis GA. Echinococcus granulosus in childhood: a retrospective study of 187 cases and newer data. Clin Pediatr (Phila) 2019;58:864-8. [CrossRef]

15. Çobanoğlu U, Sayır F, Mergan D. Diagnostic dilemma: analysis of 11 cases of hydatid disease. Turkiye Parazitol Derg 2011;35:164-8. [CrossRef]

16. Shaw JA, Irusen EM, Diacon AH, Koegelenberg CF. Pleural tuberculosis: A concise clinical review. Clin Respir J 2018;12:1779-86. [CrossRef]

17. Cruz AT, Ong LT, Starke JR. Childhood pleural tuberculosis: a review of 45 cases. Pediatr Infect Dis J 2009;28:981-4. [CrossRef]

18. Bayhan GI, Sayir F, Tanir G, Tuncer O. Pediatric pleural tuberculosis. Int J Mycobacteriol 2018;7:261-4. [CrossRef]

19. Wang JL, Zhao GW, Zhang ZQ, Wang XF, Wang MS. Clinicopathologic characteristics of pediatric tuberculous pleural effusion: a retrospective analysis of 112 consecutive cases. Eur Rev Med Pharmacol Sci 2015;19:2978-82. [CrossRef] 